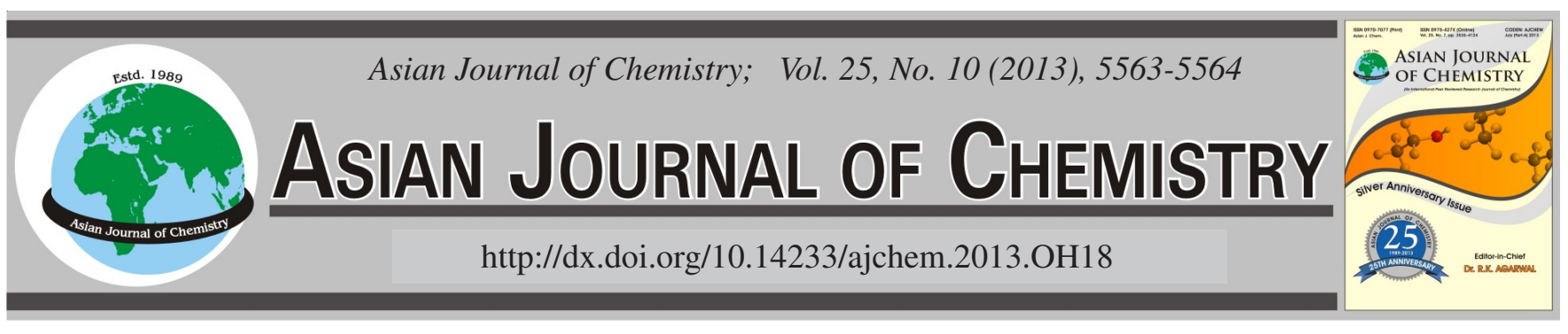

\title{
Researches on Surface of Near-field Optical Probe Based on Chemical Etching $\dagger$
}

\author{
Z.F. WANG ${ }^{1, *}$ and C.X. $\mathrm{LI}^{2}$
}

${ }^{1}$ School of Materials Science and Engineering, Anhui University of Science and Technology, Huainan, Anhui Province, P.R. China

${ }^{2}$ Medical College, Anhui University of Science and Technology, Huainan, Anhui Province, P.R. China

*Corresponding author: Tel./Fax: +82 531 6668649; E-mail: zfwang@ aust.edu.cn

Near-field scanning optical microscopy probes are produced according to classic Turner method. Temperature and etching system composed of different coverlayer and etchant mainly influence the fabrication of optical fiber probe in two ways: one is the etching velocity and the other is the change in the properties of the etching system. The diffusion of etchant in coverlayer can also affect the probes in the cone angle and yield. Probes fabricated after classic Turner method leads to rough surface of the taper, which is responsible for the formation of probe occurring at the interface of etchant and coverlayer.

Key Words: Probe, Chemical etching, Cone angle.

L

\section{INTRODUCTION}

The desire to exceed the limitations of far-field optics and to greatly improve optical resolution has led to the development of near-field scanning optical microscopy ${ }^{1-3}$. The detailed characteristics of the near-field probe play a dominant role in determining spatial resolution and signal levels in all nearfield scanning optical microscopy experiments. Numbers of properties determine the utility of a particular probe for highresolution near-field scanning optical microscopy image ${ }^{4}$. Micropipette and rob-shaped quartz crystals ${ }^{1,5,6}$ were modified for a near-field scanning optical microscopy probe and then optical fiber was adopted for convenience ${ }^{7}$. Optical fiber probes can be made by adopting chemical etching ${ }^{8-11}$ or "heating and pulling" technique ${ }^{7}$. Generally, the probes made by adopting etching method possess bigger cone angle and higher light throughput (about $10^{-4}$ ) than those made by adopting heating and pulling method. In this paper, near-field scanning optical microscopy probe was fabricated by Turner method ${ }^{11}$, different factors such as time, temperature and the concentration of etchant that influence the final probe shape and surface in our improved method were discussed.

\section{EXPERIMENTAL}

Single mode fiber with fiber's cladding diameter of $125 \mu \mathrm{m}$ and inner core diameter of $9 \mu \mathrm{m}$ (QUP-006-BXM) has been used for preparing probe tips. The fiber ends were denuded by chemical dissolution of the polyacylate protection layer in dichloromethane or chloroform.
Etching experiments were carried out at room temperatures of $20-25^{\circ} \mathrm{C}$ based on etchant covered by dimethicone, which is used to protect the optical fibers from acid vapours. Commercially available dimethicone was used without further purification. Etching was carried out in Teflon vessel. The denuded optical fibers were inserted into the corrosive solution and were kept perpendicular to the liquid interface. Scanning electron microscopy is used for determining the tip shapes and the roughness of the non-metallized horizontally-oriented fiber tips.

\section{RESULTS AND DISCUSSION}

Fig. 1 shows the SEM images of fiber probes fabricated by two different etchant A: $40 \% \mathrm{HF}$ and B: buffer etchant $40 \%$ $\mathrm{NH}_{4} \mathrm{~F}: 40 \% \mathrm{HF}: \mathrm{H}_{2} \mathrm{O}=1.7: 1: 1$. It can be seen that different cone angles have been got. The probe with even rougher surface was fabricated in a higher concentration etchant. Etching velocity was remarkably affected by temperature. Etching time was respectively $130 \mathrm{~min}$ at $0{ }^{\circ} \mathrm{C}, 70 \mathrm{~min}$ at $20^{\circ} \mathrm{C}$ and $50 \mathrm{~min}$ at $32{ }^{\circ} \mathrm{C}$ in $40 \% \mathrm{HF}$ aqueous solution. It was comprehensible that the etching velocity changed following the concentration of the etchant and temperature. The cone angle of probe was also influenced by the concentration and temperature, because the height of the meniscus depended on the characters of etchant and coverlayer, such as surface tension density and the angle between the meniscus and horizontal line ${ }^{12}$.

Fig. 2 shows taper change with time after the etching process finished in the etchant composed of $40 \% \mathrm{HF}$ and

$\lceil$ Presented to the 6th China-Korea International Conference on Multi-functional Materials and Application, 22-24 November 2012, Daejeon, Korea 
coverlayer. The optical fibers disappeared in the etchant which was etching process end (Fig. 2A). The probe tips would be etched by diffuse micro-etchant in the coverlayer if they were kept in the coverlayer ${ }^{13}$. Probes with larger cone would be prepared when probe tips were kept in a suitable time in coverlayer (Fig. 2B), but inferior fiber probes were selfinvited if prolonged time in excess (Fig. 2C). It seems that probes with larger cone could be easily fabricated by keeping the probe tips in coverlayer. However the method resulted in low fields, for the etchant diffusion was unstable and would be easily influenced by environment condition.
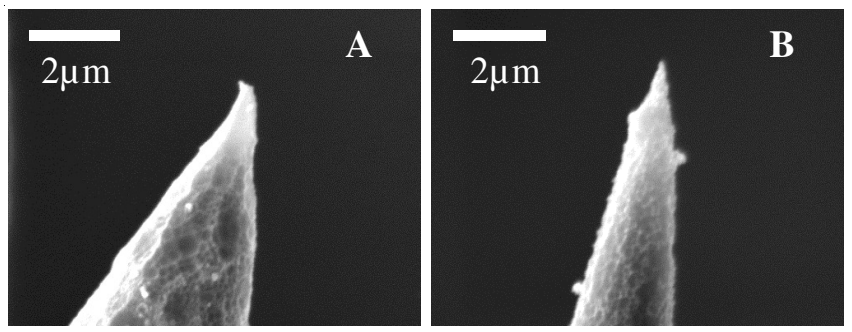

Fig. 1. SEM images of optical fibers taper A: $40 \% \mathrm{HF}$; B: buffer solution
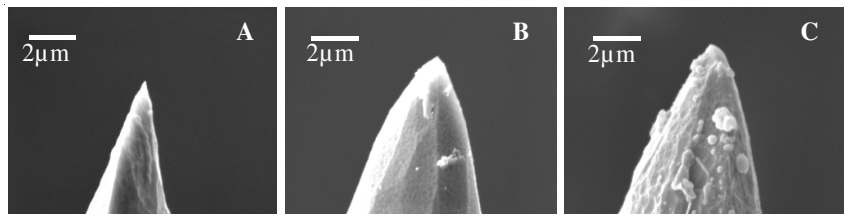

Fig. 2. SEM images of optical fiber taper changing A: $0 \mathrm{~min}$; B: $10 \mathrm{~min}$; C: $20 \mathrm{~min}$

Optical fiber probes fabricated according to classic Turner method lead to tough surface (Fig. 3), which is responsible for fact that the fibers at the interface of etchant and coverlayer were etched by etchant, thus leading to the fiber diameter lessening. The taper was formed due to decreasing meniscus height, which was influenced by the fiber diameter ${ }^{12}$. By following the interface falling down along the fiber, fiber immersed in etchant would get away the etchant layer and came into the coverlayer. There was competitive wetting action between etchant and oil during this proceeding. The etchant film covered by oil would be adsorbed on the fiber taper. The etchant in oil would be prone to form droplet, which etched the fiber taper until HF use up. This uneven etching led to a rough surface like honeycomb (Fig. 3). The more the etchant contained in droplet, the rougher surface was got. That was why probe with more rough surface was fabricated in etchant with a higher concentration, as shown in Fig. 1.
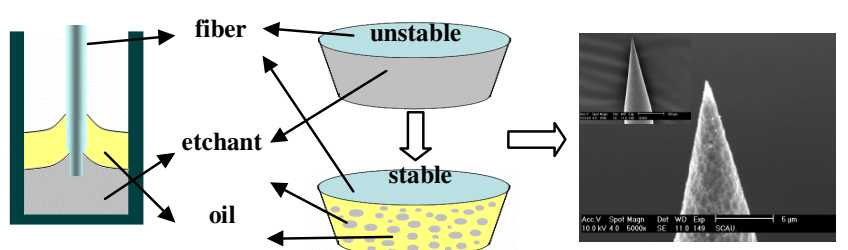

Fig. 3. Schematic of optical fiber probe with rough surface fabricated by classic Turner method and SEM images of probe tips

\section{Conclusion}

The preparation of optical probes after classic Turner method is characterized by convenience, low price and reproduction. Protection layer chemical etching was a versatile simple self-terminating method. Rough taper surface was inherent shackle with probes fabricated by classic Turner method because the taper was formed at the interface of etchant and coverlayer.

\section{ACKNOWLEDGEMENTS}

This work was financially supported by the Young Teachers Science Foundation of Anhui University of Science and Technology.

\section{REFERENCES}

1. K. Lieberman, S. Harush, A. Lewis and R. Kopelman, Science, 247, 59 (1990).

2. Trautman, J. Macklin, L. Brus and E. Betzig, Nature, 369, 40 (1994).

3. E. Betzig and R.J. Chichester, Science, 262, 1422 (1993).

4. R. Williamson and M. Miles, J. Appl. Phys., 80, 4804 (1996).

5. S. Münster, S. Werner, C. Mihalcea, W. Scholz and E. Oesterschulze, J. Micros., 186, 17 (1997).

6. K. Lieberman and A. Lewis, Appl. Phys. Lett., 62, 1335 (1993).

7. E. Betzig, J. Trautman, T. Harris, J. Weiner and R. Kostelak, Science, 251, 1468 (1991).

8. Y.H. Chuang, K.G. Sun, C.J. Wang, J. Huang and C.L. Pan, Rev. Sci. Instrum., 69, 437 (1998).

9. L. Haber, R. Schaller, J. Johnson and R. Saykally, J. Micros., 214, 27 (2004).

10. V. Machavaram, R. Badcock and G. Fernando, Sens. Actuators A, 138, 248 (2007).

11. D.R. Turner, Etch Procedure for Optical Fibers, US Patent 4469554 (1984).

12. P.K. Wong, T.H. Wang and C.M. Ho, Solid-State Sensor, Actuator and Microsystems Workshop Hilton Head Island, South Carolina, USA, (2002).

13. W. Yihong, Y. Zhiyong, Y. Tan, G. Ning and K. Wesche, J. Nanosci. Nanotechnol., 6, 1185 (2006). 